\title{
BOHMIAN PARTICLE TRAJECTORIES IN RELATIVISTIC FERMIONIC QUANTUM FIELD THEORY
}

\author{
Hrvoje Nikolić \\ Theoretical Physics Division \\ Rudjer Bošković Institute \\ P.O.B. 180, HR-10002 Zagreb, Croatia \\ E-mail: hrvoje@thphys.irb.hr
}

\begin{abstract}
The de Broglie-Bohm interpretation of quantum mechanics and quantum field theory is generalized in such a way that it describes trajectories of relativistic fermionic particles and antiparticles and provides a causal description of the processes of their creation and destruction. A general method of causal interpretation of quantum systems is developed and applied to a causal interpretation of fermionic quantum field theory represented by c-number valued wave functionals.
\end{abstract}

Key words: de Broglie-Bohm interpretation, particle trajectory, fermion, quantum field theory.

\section{INTRODUCTION}

The de Broglie-Bohm (dBB) interpretation of quantum mechanics (QM) and quantum field theory (QFT) 1, 2, 3, 4, 5, was invented with the intention to provide a causal interpretation of the conventional QM and QFT. Yet, the current form of the dBB interpretation of relativistic fermions still does not achieve this goal. First, the Dirac spinor is interpreted as a guiding wave only for one kind of corpuscles, making no difference between particle and antiparticle trajectories [6, 7, 5]. Second, QFT is not a part of the current form of the dBB interpretation of fermions. There exists a causal interpretation of fermionic fields in terms of quantum rotators 8 , [5], but this interpretation works only for free fermionic fields. Besides, the causal interpretation of fermionic fields and the interpretation in terms of causal trajectories of corpuscles are viewed as two mutually incompatible interpretations. A recent approach [9], based on earlier work [10, 11], partially resolves these problems, but still makes no difference between particle and antiparticle trajectories.

A solution of similar problems for bosons have recently been proposed in Ref. [12. In that paper, particle trajectories are determined by using the concept of particle current [13, 14, 15, and multiparticle wave functions that result from QFT. A causal interpretation of particle creation and destruction is given in terms of causally evolving fields and new hidden variables - particle effectivities. Both the particle positions and the fields are considered as beables (i.e., elements of reality). The statistical predictions of the theory are compatible with those of the standard bosonic QFT. However, despite the compatibility, the predictions of the theory are not equivalent to those of the standard bosonic QFT. Instead, the theory has predictions on the statistical distribution of particle positions, on which, in general, the standard bosonic QFT does not have clear predictions.

In this paper we generalize the results of Ref. [12 to fermionic fields. It should be considered 
mainly as a technical generalization. Consequently, the interpretational issues are not discussed in detail as they are already extensively discussed in Ref. [12]. In Sec. 2] we give a causal interpretation of the Dirac equation in terms of particle and antiparticle trajectories. In Sec. 3 we use the conventional interacting fermionic QFT in the Schrödinger picture [16, 17, 18, to construct multiparticle wave functions and the equations for the corresponding particle and antiparticle trajectories. In Sec. 4, to each fermionic state represented by a Grassmann valued wave functional we attribute a corresponding c-number valued wave functional. In Sec. 5 we develop a general method of causal interpretation of an arbitrary quantum system in which a c-number valued wave function satisfies a Schrödinger equation. In Sec. 6] we use the results of Secs. 4 and 5 to construct a causal interpretation of fermionic QFT and a causal interpretation of particle creation and destruction, in a way similar to that for bosonic fields. The conclusions are drawn in Sec. 7 .

\section{CAUSAL INTERPRETATION OF THE DIRAC EQUATION}

The Dirac equation in the Minkowski metric $\eta_{\mu \nu}=\operatorname{diag}(1,-1,-1,-1)$ is

$$
\left(i \gamma^{\mu} \partial_{\mu}-m\right) \psi(x)=0,
$$

where $x=\left(x^{0}, x^{1}, x^{2}, x^{3}\right)=(t, \mathbf{x})$. The general solution of (10) can be written as

$$
\psi(x)=\psi^{(P)}(x)+\psi^{(A)}(x),
$$

where the particle and antiparticle parts can be expanded as

$$
\psi^{(P)}(x)=\sum_{k} b_{k} u_{k}(x), \quad \psi^{(A)}(x)=\sum_{k} d_{k}^{*} v_{k}(x),
$$

respectively. Here $u_{k}\left(v_{k}\right)$ are positive (negative) frequency 4-spinors that, together, form a complete orthonormal set of solutions to (1). The label $k$ is an abbreviation for the set $(\mathbf{k}, s)$, where $\mathbf{k}$ is a 3 -vector related to a Fourier expansion and $s= \pm 1 / 2$ is the spin label.

The interpretation of negative frequency spinors as antiparticles (i.e., positrons) above is usually considered as a part of second quantization, i.e., QFT. Indeed, QFT is discussed in the next section. However, by using the Stückelberg-Feynman theory, such an interpretation is meaningful even without explicit introduction of QFT [19].

By introducing the quantities

$$
\begin{aligned}
& \Omega^{(P)}\left(x, x^{\prime}\right)=\sum_{k} u_{k}(x) u_{k}^{\dagger}\left(x^{\prime}\right), \\
& \Omega^{(A)}\left(x, x^{\prime}\right)=\sum_{k} v_{k}(x) v_{k}^{\dagger}\left(x^{\prime}\right),
\end{aligned}
$$

we can extract $\psi^{(P)}$ and $\psi^{(A)}$ from $\psi$ using

$$
\begin{aligned}
& \psi^{(P)}(x)=\int d^{3} x^{\prime} \Omega^{(P)}\left(x, x^{\prime}\right) \psi\left(x^{\prime}\right), \\
& \psi^{(A)}(x)=\int d^{3} x^{\prime} \Omega^{(A)}\left(x, x^{\prime}\right) \psi\left(x^{\prime}\right),
\end{aligned}
$$

where $t=t^{\prime}$. We introduce the particle and antiparticle currents defined as

$$
j_{\mu}^{(P)}=\bar{\psi}^{(P)} \gamma_{\mu} \psi^{(P)}, \quad j_{\mu}^{(A)}=\bar{\psi}^{(A)} \gamma_{\mu} \psi^{(A)},
$$


respectively, where $\bar{\psi}=\psi^{\dagger} \gamma_{0}$. Since $\psi^{(P)}$ and $\psi^{(A)}$ separately satisfy the Dirac equation (11), the currents $j_{\mu}^{(P)}$ and $j_{\mu}^{(A)}$ are separately conserved: $\partial^{\mu} j_{\mu}^{(P)}=\partial^{\mu} j_{\mu}^{(A)}=0$. Therefore, we postulate that trajectories of particles and antiparticles are given by

$$
\frac{d \mathbf{x}^{(P)}}{d t}=\frac{\mathbf{j}^{(P)}\left(t, \mathbf{x}^{(P)}\right)}{j_{0}^{(P)}\left(t, \mathbf{x}^{(P)}\right)}, \quad \frac{d \mathbf{x}^{(A)}}{d t}=\frac{\mathbf{j}^{(A)}\left(t, \mathbf{x}^{(A)}\right)}{j_{0}^{(A)}\left(t, \mathbf{x}^{(A)}\right)},
$$

respectively, where $\mathbf{j}=\left(j^{1}, j^{2}, j^{3}\right)$.

\section{PARTICLE AND ANTIPARTICLE TRAJECTORIES IN FERMIONIC QFT}

In fermionic QFT, the coefficients $b_{k}$ and $d_{k}^{*}$ in (3) become anticommuting operators. The operators $\hat{b}_{k}^{\dagger}\left(\hat{d}_{k}^{\dagger}\right)$ create particles (antiparticles), while $\hat{b}_{k}$ and $\hat{d}_{k}$ annihilate them. In the Schrödinger picture, the field operators $\hat{\psi}(\mathbf{x})$ and $\hat{\psi}^{\dagger}(\mathbf{x})$ satisfy the anticommutation relations

$$
\left\{\hat{\psi}_{a}(\mathbf{x}), \hat{\psi}_{a^{\prime}}^{\dagger}\left(\mathbf{x}^{\prime}\right)\right\}=\delta_{a a^{\prime}} \delta^{3}\left(\mathbf{x}-\mathbf{x}^{\prime}\right),
$$

while other anticommutators vanish. (Here $a$ is a spinor index.) These anticommutation relations can be represented by

$$
\begin{aligned}
& \hat{\psi}_{a}(\mathbf{x})=\frac{1}{\sqrt{2}}\left[\eta_{a}(\mathbf{x})+\frac{\delta}{\delta \eta_{a}^{*}(\mathbf{x})}\right], \\
& \hat{\psi}_{a}^{\dagger}(\mathbf{x})=\frac{1}{\sqrt{2}}\left[\eta_{a}^{*}(\mathbf{x})+\frac{\delta}{\delta \eta_{a}(\mathbf{x})}\right],
\end{aligned}
$$

where $\eta_{a}(\mathbf{x})$ and $\eta_{a}^{*}(\mathbf{x})$ are anticommuting Grassmann numbers:

$$
\left\{\eta_{a}(\mathbf{x}), \eta_{a^{\prime}}\left(\mathbf{x}^{\prime}\right)\right\}=\left\{\eta_{a}^{*}(\mathbf{x}), \eta_{a^{\prime}}^{*}\left(\mathbf{x}^{\prime}\right)\right\}=\left\{\eta_{a}(\mathbf{x}), \eta_{a^{\prime}}^{*}\left(\mathbf{x}^{\prime}\right)\right\}=0 .
$$

Next we introduce a complete orthonormal set of spinors $u_{k}(\mathbf{x})$ and $v_{k}(\mathbf{x})$. It is convenient to choose them to be equal to the spinors $u_{k}(x)$ and $v_{k}(x)$ at $t=0$, respectively. An arbitrary quantum state may be obtained by acting with the creation operators

$$
\begin{aligned}
& \hat{b}_{k}^{\dagger}=\int d^{3} x \hat{\psi}^{\dagger}(\mathbf{x}) u_{k}(\mathbf{x}), \\
& \hat{d}_{k}^{\dagger}=\int d^{3} x v_{k}^{\dagger}(\mathbf{x}) \hat{\psi}(\mathbf{x}),
\end{aligned}
$$

on the vacuum $|0\rangle \equiv\left|\Psi_{0}\right\rangle$ represented by

$$
\Psi_{0}\left[\eta, \eta^{\dagger}\right]=N \exp \left\{\int d^{3} x \int d^{3} x^{\prime} \eta^{\dagger}(\mathbf{x}) \Omega\left(\mathbf{x}, \mathbf{x}^{\prime}\right) \eta\left(\mathbf{x}^{\prime}\right)\right\} .
$$

Here $\Omega\left(\mathbf{x}, \mathbf{x}^{\prime}\right)=\Omega^{(A)}\left(\mathbf{x}, \mathbf{x}^{\prime}\right)-\Omega^{(P)}\left(\mathbf{x}, \mathbf{x}^{\prime}\right), N$ is a constant chosen such that $\left\langle\Psi_{0} \mid \Psi_{0}\right\rangle=1$ and the scalar product is

$$
\left\langle\Psi \mid \Psi^{\prime}\right\rangle=\int \mathcal{D}^{2} \eta \Psi^{*}\left[\eta, \eta^{\dagger}\right] \Psi^{\prime}\left[\eta, \eta^{\dagger}\right]
$$

In (13), $\mathcal{D}^{2} \eta \equiv \mathcal{D} \eta \mathcal{D} \eta^{\dagger}$ and $\Psi^{*}$ is dual (not simply the complex conjugate [16, 17]) to $\Psi$. The vacuum is chosen such that $\hat{b}_{k} \Psi_{0}=\hat{d}_{k} \Psi_{0}=0$. A functional $\Psi\left[\eta, \eta^{\dagger}\right]$ can be expanded as

$$
\Psi\left[\eta, \eta^{\dagger}\right]=\sum_{K} c_{K} \Psi_{K}\left[\eta, \eta^{\dagger}\right],
$$


where the set $\left\{\Psi_{K}\right\}$ is a complete orthonormal set of Grassmann valued functionals. We choose this set such that each $\Psi_{K}$ is proportional to a functional of the form $\hat{b}_{k_{1}}^{\dagger} \cdots \hat{b}_{k_{n_{P}}}^{\dagger} \hat{d}_{k_{1}^{\prime}}^{\dagger} \cdots \hat{d}_{k_{n_{A}}^{\prime}}^{\dagger} \Psi_{0}$, which means that each $\Psi_{K}$ has a definite number $n_{P}$ of particles and a definite number $n_{A}$ of antiparticles. Therefore, we can also write (14) as

$$
\Psi\left[\eta, \eta^{\dagger}\right]=\sum_{n_{P}, n_{A}=0}^{\infty} \tilde{\Psi}_{n_{P}, n_{A}}\left[\eta, \eta^{\dagger}\right] .
$$

The tilde on $\tilde{\Psi}_{n_{P}, n_{A}}$ denotes that these functionals, in contrast with $\Psi$ and $\Psi_{K}$, do not have unit norm.

Time-dependent states $\Psi\left[\eta, \eta^{\dagger}, t\right]$ can be expanded as

$$
\begin{aligned}
\Psi\left[\eta, \eta^{\dagger}, t\right] & =\sum_{K} c_{K}(t) \Psi_{K}\left[\eta, \eta^{\dagger}\right] \\
& =\sum_{n_{P}, n_{A}=0}^{\infty} \tilde{\Psi}_{n_{P}, n_{A}}\left[\eta, \eta^{\dagger}, t\right] .
\end{aligned}
$$

The time dependence of the c-number coefficients $c_{K}(t)$ is governed by the functional Schrödinger equation

$$
H\left[\hat{\psi}, \hat{\psi}^{\dagger}\right] \Psi\left[\eta, \eta^{\dagger}, t\right]=i \partial_{t} \Psi\left[\eta, \eta^{\dagger}, t\right] .
$$

Since the Hamiltonian $H$ is a hermitian operator, the norms

$$
\langle\Psi(t) \mid \Psi(t)\rangle=\sum_{K}\left|c_{K}(t)\right|^{2}
$$

of the states do not depend on time. In particular, if $H$ is the free Hamiltonian (i.e. the Hamiltonian that generates the second quantized free Dirac equation (10), then the quantities $\left|c_{K}(t)\right|$ do not depend on time. This means that the average number of particles and antiparticles does not change with time when the interactions are absent.

Next we introduce the wave function of $n_{P}$ particles and $n_{A}$ antiparticles, denoted as

$$
\psi_{n_{P}, n_{A}} \equiv \psi_{b_{1} \cdots b_{n_{P}} d_{1} \cdots d_{n_{A}}}\left(\mathbf{x}_{1}, \ldots, \mathbf{x}_{n_{P}}, \mathbf{y}_{1}, \ldots, \mathbf{y}_{n_{A}}, t\right) .
$$

It has $n_{P}+n_{A}$ spinor indices $b_{1}, \cdots, d_{n_{A}}$. For free fields, the (unnormalized) wave function can be calculated using the Heisenberg picture as

$$
\psi_{n_{P}, n_{A}}=\left\langle 0\left|\hat{\psi}_{b_{1}}^{(P)}\left(t, \mathbf{x}_{1}\right) \cdots \hat{\psi}_{d_{n_{A}}}^{(A) \dagger}\left(t, \mathbf{y}_{n_{A}}\right)\right| \Psi\right\rangle,
$$

where $\hat{\psi}^{(P)}$ and $\hat{\psi}^{(A)}$ are extracted from $\hat{\psi}$ with the aid of (5). In the general, interacting case, the wave function can be calculated using the Schrödinger picture as

$$
\begin{aligned}
\psi_{n_{P}, n_{A}}= & \int \mathcal{D}^{2} \eta \Psi_{0}^{*}\left[\eta, \eta^{\dagger}\right] e^{-i \varphi_{0}(t)} \\
& \times \hat{\psi}_{b_{1}}^{(P)}\left(\mathbf{x}_{1}\right) \cdots \hat{\psi}_{d_{n_{A}}}^{(A) \dagger}\left(\mathbf{y}_{n_{A}}\right) \Psi\left[\eta, \eta^{\dagger}, t\right] .
\end{aligned}
$$

Here the phase $\varphi_{0}(t)$ is defined by an expansion of the form of (16):

$$
\hat{U}(t) \Psi_{0}\left[\eta, \eta^{\dagger}\right]=r_{0}(t) e^{i \varphi_{0}(t)} \Psi_{0}\left[\eta, \eta^{\dagger}\right]+\sum_{\left(n_{P}, n_{A}\right) \neq(0,0)} \ldots,
$$

where $r_{0}(t) \geq 0$ and $\hat{U}(t)=U\left[\hat{\psi}, \hat{\psi}^{\dagger}, t\right]$ is the unitary time-evolution operator that satisfies the Schrödinger equation (17). Note that only $\tilde{\Psi}_{n_{P}, n_{A}}$ from the expansion (16) contributes to (21). 
The current attributed to the $i$-th corpuscle (particle or antiparticle) in the wave function $\psi_{n_{P}, n_{A}}$ is

$$
j_{\mu(i)}= \begin{cases}\bar{\chi} \gamma_{\mu(i)} \chi & \text { for particles } \\ \bar{\chi} \gamma_{\mu(i)}^{T} \chi & \text { for antiparticles }\end{cases}
$$

where we have used abbreviations

$$
\begin{aligned}
& \bar{\chi} \Gamma_{(i)} \chi \equiv \bar{\chi}_{a_{1} \cdots a_{i} \cdots a_{n}}(\Gamma)_{a_{i} a_{i}^{\prime}} \chi_{a_{1} \cdots a_{i}^{\prime} \cdots a_{n}} \\
& \bar{\chi}_{b_{1} \cdots b_{n_{P}} d_{1} \cdots d_{n_{A}}} \equiv \psi_{b_{1}^{\prime} \cdots b_{n_{P}}^{\prime} d_{1} \cdots d_{n_{A}}}^{*}\left(\gamma_{0}\right)_{b_{1}^{\prime} b_{1}} \cdots\left(\gamma_{0}\right)_{b_{n_{P}}^{\prime} b_{n_{P}}}, \\
& \chi_{b_{1} \cdots b_{n_{P}} d_{1} \cdots d_{n_{A}}} \equiv\left(\gamma_{0}^{T}\right)_{d_{1} d_{1}^{\prime}} \cdots\left(\gamma_{0}^{T}\right)_{d_{n_{A}} d_{n_{A}}^{\prime}} \psi_{b_{1} \cdots b_{n_{P}} d_{1}^{\prime} \cdots d_{n_{A}}^{\prime}}^{*} \text {, }
\end{aligned}
$$

and the superscript $T$ denotes the transpose. Eq. (23) is the generalization of (6) for multiparticle wave functions. Therefore, the trajectory of the $i$-th corpuscle guided by the wave function $\psi_{n_{P}, n_{A}}$ is given by the generalization of (7)

$$
\frac{d \mathbf{x}_{(i)}}{d t}=\frac{\mathbf{j}_{(i)}}{j_{0(i)}} .
$$

As an example, consider the wave function describing one positron

$$
\psi_{a}^{(A) *}(x)=\psi_{a}(x)=\left\langle 0\left|\hat{\psi}_{a}^{(A) \dagger}(x)\right| \Psi\right\rangle .
$$

From (25) we find $\chi=\gamma_{0}^{T} \psi, \bar{\chi}=\psi^{\dagger}$, so (23) reduces to

$$
j_{\mu}^{(A)}=\psi^{\dagger} \gamma_{\mu}^{T} \gamma_{0}^{T} \psi=\psi^{(A) \dagger} \gamma_{0} \gamma_{\mu} \psi^{(A)}=\bar{\psi}^{(A)} \gamma_{\mu} \psi^{(A)} .
$$

Note that the wave function that corresponds to the presence (not the absence) of a positron is $\psi_{a}^{(A) *}\left(\right.$ not $\left.\psi_{a}^{(A)}\right)$. In this sense, we were slightly imprecise in Sec. 2 by referring to $\psi^{(A)}$ as the antiparticle part. Nevertheless, the expression (6) for the antiparticle current is consistent with (28). The wave function (27) satisfies the same equation of motion (Dirac equation) as the field operator $\hat{\psi}_{a}^{(A) \dagger}(x)$. Consequently, the current (28) is conserved

$$
\partial^{\mu} j_{\mu}^{(A)}=0
$$

and naturally interpreted as the positron current. In particular, the quantity $j_{0}^{(A)}=\psi_{a}^{(A) *} \psi_{a}^{(A)}$ is naturally interpreted as the positron density, which can be demonstrated as follows. The operator of the positron density is [15]

$$
\hat{n}^{(A)}(x)=\hat{\psi}_{a}^{(A)}(x) \hat{\psi}_{a}^{(A) \dagger}(x) .
$$

Let $|\Psi\rangle$ be a free one positron state

$$
|\Psi\rangle=\sum_{k} c_{k} \hat{d}_{k}^{\dagger}|0\rangle
$$

where $c_{k}$ are some coefficients. The corresponding wave function is given by the complex conjugate of

$$
\psi^{(A)}(x)=\sum_{k} c_{k}^{*} v_{k}(x),
$$


while the expectation value of the positron density is

$$
\left\langle\Psi\left|\hat{n}^{(A)}(x)\right| \Psi\right\rangle=\psi_{a}^{(A) *}(x) \psi_{a}^{(A)}(x) .
$$

Note that the positron wave function is not a coefficient of a decomposition of a quantum-field state $|\Psi\rangle$ in eigenstates of the positron density operator. In the conventional interpretation of QFT, this would imply that the positron density cannot be interpreted as the positron probability density. Indeed, the conventional interpretation of QFT does not have definite predictions on the probabilities of particle and antiparticle positions [12. However, similarly to the bosonic case [12, our interpretation of fermions is an extension of the conventional interpretation. The extension consists in postulating the particle and antiparticle trajectories. Eq. (26), together with the conservation equation (29), provides the consistency of the probabilistic interpretation, i.e., the equivariance. If the positrons in a statistical ensemble are initially distributed with probabilities equal to $\psi_{a}^{(A) *} \psi_{a}^{(A)}$, then (26) and (29) imply that the positrons will be distributed according to $\psi_{a}^{(A) *} \psi_{a}^{(A)}$ at all times.

It is also important to emphasize that, similarly to the bosonic case [12, our extension is compatible with the conventional interpretation in the sense that any definite prediction of the conventional interpretation is also a prediction of our interpretation. With our extension, we do not deny any standard QFT effect that can be described purely in terms of quantum fields without explicit introduction of the concept of particles. In particular, although our extension does not attribute a particle trajectory to the vacuum, the standard measurable effects of the vacuum are not denied by our extension. The set of all predictions of the conventional interpretation is a subset of the set of all predictions of our interpretation.

\section{BOSONIZATION OF FERMIONIC QFT}

So far, we have not introduced any causal interpretation of the fermionic fields $\hat{\psi}$ or $\eta$. Actually, these two quantities are not observables, so there is no need for a causal interpretation of them. However, we need a causal interpretation of the processes of creation and destruction of particles and antiparticles. For bosonic fields, this can be achieved by introducing a new causally evolving hidden variable - the effectivity of a particle [12] - determined by the causal evolution of bosonic fields. Unfortunately, one cannot introduce a similar causal theory for the Grassmann fields $\eta$ and $\eta^{\dagger}$, essentially because the quantity $\Psi^{*}\left[\eta, \eta^{\dagger}, t\right] \Psi\left[\eta, \eta^{\dagger}, t\right]$ is Grassmann valued, so, unlike to the bosonic case, one cannot interpret this quantity as a probability density. Therefore, in this section we formulate another representation of fermionic states, more similar to that of bosonic states. In Sec. 6, using the general method developed in Sec. 5], we use this representation to postulate a causal interpretation of fermionic QFT and a causal interpretation of the processes of creation and destruction of particles and antiparticles, similar to that of bosonic fields.

Note first that the notion of the scalar product can be generalized in such a way that it may be Grassmann valued [18, which allows us to write $\Psi\left[\eta, \eta^{\dagger}, t\right]=\left\langle\eta, \eta^{\dagger} \mid \Psi(t)\right\rangle$ and

$$
1=\int \mathcal{D}^{2} \eta\left|\eta, \eta^{\dagger}\right\rangle\left\langle\eta, \eta^{\dagger}\right|
$$

Next, for each fermionic state $\Psi_{K}\left[\eta, \eta^{\dagger}\right]$ we introduce the corresponding bosonic state $\Psi_{K}\left[\varphi, \varphi^{\dagger}\right]$, with $\varphi(\mathbf{x})$ and $\varphi^{\dagger}(\mathbf{x})$ being c-number 4 -spinors. The bosonic states $\Psi_{K}$ are obtained in the same way as the corresponding fermionic states, by acting with the bosonic creation operators $\hat{b}_{k}^{\dagger}$ and $\hat{d}_{k}^{\dagger}$ (that satisfy the corresponding commutation relations) on the bosonic vacuum $\Psi_{0}\left[\varphi, \varphi^{\dagger}\right]$. (For details on the bosonic functional methods, see e.g. Refs. [5, 16, 18, 20]). Note that the set 
of bosonic states $\left\{\Psi_{K}\left[\varphi, \varphi^{\dagger}\right]\right\}$ does not contain states obtained by acting with the same creation operator on the vacuum more than once (e.g. $\left.\left(\hat{b}_{k}^{\dagger}\right)^{2}|0\rangle\right)$ because such states vanish identically in the fermionic case. Therefore, the set of states $\left\{\Psi_{K}\left[\varphi, \varphi^{\dagger}\right]\right\}$ is a complete orthonormal basis for a subspace of the whole space of all well-behaved functionals $\Phi\left[\varphi, \varphi^{\dagger}\right]$. On this subspace, the unit operator can be represented as

$$
1=\sum_{K}\left|\Psi_{K}\right\rangle\left\langle\Psi_{K}\right|
$$

We can also introduce the quantity

$$
\begin{aligned}
\left\langle\varphi, \varphi^{\dagger} \mid \eta, \eta^{\dagger}\right\rangle & =\sum_{K}\left\langle\varphi, \varphi^{\dagger} \mid \Psi_{K}\right\rangle\left\langle\Psi_{K} \mid \eta, \eta^{\dagger}\right\rangle \\
& =\sum_{K} \Psi_{K}\left[\varphi, \varphi^{\dagger}\right] \Psi_{K}^{*}\left[\eta, \eta^{\dagger}\right],
\end{aligned}
$$

so we see that the sets $\left\{\Psi_{K}\left[\eta, \eta^{\dagger}\right]\right\}$ and $\left\{\Psi_{K}\left[\varphi, \varphi^{\dagger}\right]\right\}$ are two representations of the same orthonormal basis $\left\{\left|\Psi_{K}\right\rangle\right\}$ for the same Hilbert space of fermionic states. In other words, the fermionic state $|\Psi(t)\rangle$ can be represented as $\Psi\left[\varphi, \varphi^{\dagger}, t\right]=\left\langle\varphi, \varphi^{\dagger} \mid \Psi(t)\right\rangle$, which, by using (34), (36) and (16), can be expanded as

$$
\begin{aligned}
\Psi\left[\varphi, \varphi^{\dagger}, t\right] & =\sum_{K} c_{K}(t) \Psi_{K}\left[\varphi, \varphi^{\dagger}\right] \\
& =\sum_{n_{P}, n_{A}=0}^{\infty} \tilde{\Psi}_{n_{P}, n_{A}}\left[\varphi, \varphi^{\dagger}, t\right] .
\end{aligned}
$$

By inserting the unit operator $1=\int \mathcal{D}^{2} \varphi\left|\varphi, \varphi^{\dagger}\right\rangle\left\langle\varphi, \varphi^{\dagger}\right|$ in (18), we see that the time-independent norm can be written as

$$
\langle\Psi(t) \mid \Psi(t)\rangle=\int \mathcal{D}^{2} \varphi \Psi^{*}\left[\varphi, \varphi^{\dagger}, t\right] \Psi\left[\varphi, \varphi^{\dagger}, t\right] .
$$

Therefore, we interpret the quantity

$$
\rho\left[\varphi, \varphi^{\dagger}, t\right]=\Psi^{*}\left[\varphi, \varphi^{\dagger}, t\right] \Psi\left[\varphi, \varphi^{\dagger}, t\right]
$$

as a positive definite probability density for spinors $\varphi$ and $\varphi^{\dagger}$ to have space dependence $\varphi(\mathbf{x})$ and $\varphi^{\dagger}(\mathbf{x})$, respectively, at time $t$.

The Schrödinger equation (17) can also be written in the $\varphi$-representation as

$$
\hat{H}_{\varphi} \Psi\left[\varphi, \varphi^{\dagger}, t\right]=i \partial_{t} \Psi\left[\varphi, \varphi^{\dagger}, t\right] .
$$

The Hamiltonian $\hat{H}_{\varphi}$ is defined by its action on wave functionals $\Psi\left[\varphi, \varphi^{\dagger}, t\right]$. This action is determined by

$$
\begin{aligned}
\hat{H}_{\varphi} \Psi\left[\varphi, \varphi^{\dagger}, t\right]= & \int \mathcal{D}^{2} \eta \int \mathcal{D}^{2} \varphi^{\prime}\left\langle\varphi, \varphi^{\dagger} \mid \eta, \eta^{\dagger}\right\rangle \\
& \times \hat{H}\left\langle\eta, \eta^{\dagger} \mid \varphi^{\prime}, \varphi^{\prime \dagger}\right\rangle \Psi\left[\varphi^{\prime}, \varphi^{\prime \dagger}, t\right],
\end{aligned}
$$

where $\hat{H}=H\left[\hat{\psi}, \hat{\psi}^{\dagger}\right]$ is the Hamiltonian in (17).

Having constructed the $\varphi$-representation of fermionic QFT, we can introduce a causal interpretation of it. We first develop a general method of causal interpretation in the next section and then apply it to fermionic QFT in Sec. [6] 


\section{GENERAL METHOD OF CAUSAL INTERPRETATION}

In this section we develop a general method of causal interpretation of a quantum system described by a c-number valued wave function that satisfies a Schrödinger equation. For simplicity, we study the case of $n$ degrees of freedom denoted by a real $n$-dimensional vector $\vec{\varphi}$, but we write all equations in a form that can be easily generalized to the case with an infinite number of the degrees of freedom.

The wave function $\psi(\vec{\varphi}, t)$ satisfies the Schrödinger equation

$$
\hat{H} \psi=i \partial_{t} \psi
$$

where $\hat{H}$ is an arbitrary hermitian Hamiltonian (written in the $\vec{\varphi}$-representation). The quantity $\rho=\psi^{*} \psi$ is the probability density for the variables $\vec{\varphi}$. The corresponding average velocity is

$$
\frac{d\langle\vec{\varphi}\rangle(t)}{d t}=\int d^{n} \varphi \rho(\vec{\varphi}, t) \vec{u}(\vec{\varphi}, t)
$$

where $\vec{u}$ is defined as

$$
\vec{u}=\operatorname{Re} i \frac{\psi^{*}[\hat{H}, \vec{\varphi}] \psi}{\psi^{*} \psi} .
$$

We introduce the source $J$ defined as

$$
J=\frac{\partial \rho}{\partial t}+\vec{\nabla}(\rho \vec{u})
$$

For many physically interesting Hamiltonians, the source $J$ vanishes. However, in general, $J$ does not need to vanish. An example for which $J \neq 0$ is provided by the Hamiltonian $\hat{H}_{\varphi}$ defined by (41). We want to find the quantity $\vec{v}(\vec{\varphi}, t)$ that has the property (43)

$$
\frac{d\langle\vec{\varphi}\rangle(t)}{d t}=\int d^{n} \varphi \rho(\vec{\varphi}, t) \vec{v}(\vec{\varphi}, t)
$$

but at the same time satisfies the equivariance property

$$
\frac{\partial \rho}{\partial t}+\vec{\nabla}(\rho \vec{v})=0
$$

These two properties allow us to postulate a consistent causal interpretation of quantum mechanics in which $\vec{\varphi}$ has definite values at each time $t$, determined by

$$
\frac{d \vec{\varphi}}{d t}=\vec{v}(\vec{\varphi}, t)
$$

In particular, the equivariance (47) provides that the statistical distribution of the variables $\vec{\varphi}$ is given by $\rho$ for any time $t$, provided that it is given by $\rho$ for some initial time $t_{0}$. When $J=0$ then $\vec{v}=\vec{u}$, which corresponds to the usual dBB interpretation. The aim of this section is to generalize this to the general case $J \neq 0$.

Let us write the velocity $\vec{v}$ in the form

$$
\vec{v}=\vec{u}+\rho^{-1} \overrightarrow{\mathcal{E}}
$$

where $\overrightarrow{\mathcal{E}}(\vec{\varphi}, t)$ is the quantity that needs to be determined. From (47), we see that $\overrightarrow{\mathcal{E}}$ must be a solution of the equation

$$
\vec{\nabla} \overrightarrow{\mathcal{E}}=-J
$$


Let $\vec{E}(\vec{\varphi}, t)$ be some particular solution of (150). Then

$$
\overrightarrow{\mathcal{E}}(\vec{\varphi}, t)=\vec{e}(t)+\vec{E}(\vec{\varphi}, t)
$$

is also a solution for an arbitrary $\vec{\varphi}$-independent function $\vec{e}(t)$. Comparing (46) with (43), we see that we must require $\int d^{n} \varphi \overrightarrow{\mathcal{E}}=0$. This fixes the function $\vec{e}(t)$ to be

$$
\vec{e}(t)=-V^{-1} \int d^{n} \varphi \vec{E}(\vec{\varphi}, t)
$$

where $V \equiv \int d^{n} \varphi$. It remains to choose the particular solution $\vec{E}$. We choose it such that $\vec{E}=0$ when $J=0$. Eqs. (52) and (51) then imply that $\overrightarrow{\mathcal{E}}=0$ when $J=0$, i.e. that $\vec{v}=\vec{u}$ when $J=0$. There is still some arbitrariness in choosing the particular solution $\vec{E}$, so we proceed in a way that seems to be the simplest one. We take $\vec{E}$ to be a gradient of a scalar function

$$
\vec{E}=\vec{\nabla} \Phi
$$

so $\Phi$ satisfies the Poisson equation

$$
\vec{\nabla}^{2} \Phi=-J
$$

It is convenient to solve (54) by using the Green-function method. By taking the solution to have the form

$$
\Phi(\vec{\varphi}, t)=\int d^{n} \varphi^{\prime} G\left(\vec{\varphi}, \vec{\varphi}^{\prime}\right) J\left(\vec{\varphi}^{\prime}, t\right)
$$

we see from (54) that $G$ satisfies

$$
\vec{\nabla}^{2} G\left(\vec{\varphi}, \vec{\varphi}^{\prime}\right)=-\delta^{n}\left(\vec{\varphi}-\vec{\varphi}^{\prime}\right) .
$$

The solution of (56) is most easily expressed as a Fourier transform

$$
G\left(\vec{\varphi}, \vec{\varphi}^{\prime}\right)=\int \frac{d^{n} k}{(2 \pi)^{n}} \frac{e^{i \vec{k}\left(\vec{\varphi}-\vec{\varphi}^{\prime}\right)}}{\vec{k}^{2}}
$$

To eliminate the factor $1 /(2 \pi)^{n}$, we introduce a new integration variable $\vec{\chi}=\vec{k} / 2 \pi$. In this way, (55) becomes

$$
\Phi(\vec{\varphi}, t)=\int d^{n} \chi \int d^{n} \varphi^{\prime} \frac{e^{i 2 \pi \vec{\chi}\left(\vec{\varphi}-\vec{\varphi}^{\prime}\right)}}{(2 \pi)^{2} \vec{\chi}^{2}} J\left(\vec{\varphi}^{\prime}, t\right),
$$

which is written in the form appropriate for a generalization to an infinite number of the degrees of freedom.

The formalism above can also be easily generalized to the case in which the integration measure in (43) is not simply $d^{n} \varphi$, but a more general expression of the form $d^{n} \varphi \mu(\vec{\varphi}, t)$. In some cases, $\mu$ is a Jacobian that can be eliminated by a redefinition of the integration variables $\vec{\varphi}$. If this is not the case, $\mu$ can always be absorbed into a redefinition of the density $\rho \rightarrow \mu \rho$.

There is one additional tacit assumption in our general analysis that does not need to be satisfied in an even more general situation: the components $\varphi_{i}$ of the vector $\vec{\varphi}$ are assumed to be continuous parameters. If these parameters were discrete instead of continuous, then Eq. (43) would contain a summation over allowed values of $\vec{\varphi}$ instead of the integration over them. Examples of discrete variables are the $z$-component of spin and the momentum of a particle confined in a finite volume. There exists a consistent causal interpretation for these two examples [5], but it is not clear how to introduce a general systematic method of causal interpretation of discrete quantum variables. Formally, one might reduce the summation to an integration by introducing the integration measure in which the function $\mu$ is essentially a sum of 
$\delta$-functions. However, owing to the singular nature of $\delta$-functions, it is not clear that this would lead to a mathematically well-defined causal theory (see e.g. Ref. 21]). A recent approach [22] suggests a solution of this problem based on smearing of the $\delta$-functions, but this approach does not seem to be unique. Therefore, the formulation of a general method of causal interpretation of discrete quantum variables is still a challenge. Fortunately, we do not need it in this paper.

\section{CAUSAL INTERPRETATION OF FERMIONIC QFT}

We are now ready to formulate a causal interpretation of fermionic QFT. In Sec. 4 we have been working with complex spinors $\varphi$ and $\varphi^{\dagger}$, having complex components $\varphi_{a}$ and $\varphi_{a}^{*}$, respectively. On the other hand, in Sec. [5 we have developed a general method of causal interpretation for real variables. Therefore, in this section we first introduce real spinor components $\varphi_{a}^{1}$ and $\varphi_{a}^{2}$ defined by $\varphi_{a}=\left(\varphi_{a}^{1}+i \varphi_{a}^{2}\right) / \sqrt{2}$, so that

$$
\varphi_{a}^{1}=\frac{\varphi_{a}^{*}+\varphi_{a}}{\sqrt{2}}, \quad \varphi_{a}^{2}=i \frac{\varphi_{a}^{*}-\varphi_{a}}{\sqrt{2}} .
$$

Each functional of the complex spinors $\varphi$ and $\varphi^{\dagger}$ can also be viewed as a functional of the real spinors $\varphi^{1}$ and $\varphi^{2}$. To simplify the notation, when appropriate, we denote the functionals of the form of $A\left[\varphi^{1}, \varphi^{2}, t, \mathbf{x}\right]$ by a shorter simbol $A[\mathbf{x}]$.

Following the general method developed in Sec. 5 , we introduce the quantity

$$
u_{a}^{r}[\mathbf{x}]=\operatorname{Re} i \frac{\Psi^{*}\left[\hat{H}_{\varphi}, \varphi_{a}^{r}(\mathbf{x})\right] \Psi}{\Psi^{*} \Psi}
$$

where $r=1,2$ and $\Psi=\Psi\left[\varphi^{1}, \varphi^{2}, t\right]$. Next, we introduce the source

$$
J=\frac{\partial \rho}{\partial t}+\sum_{a, r} \int d^{3} x \frac{\delta\left(\rho u_{a}^{r}[\mathbf{x}]\right)}{\delta \varphi_{a}^{r}(\mathbf{x})}
$$

where $\rho=\Psi^{*} \Psi$. Introducing the notation

$$
\alpha \cdot \beta \equiv \sum_{a, r} \int d^{3} x \alpha_{a}^{r}(\mathbf{x}) \beta_{a}^{r}(\mathbf{x}),
$$

Eq. (58) generalizes to

$$
\Phi\left[\varphi^{1}, \varphi^{2}, t\right]=\int \mathcal{D}^{2} \chi \int \mathcal{D}^{2} \varphi^{\prime} \frac{e^{i 2 \pi \chi \cdot\left(\varphi-\varphi^{\prime}\right)}}{(2 \pi)^{2} \chi \cdot \chi} J\left[\varphi^{\prime 1}, \varphi^{\prime 2}, t\right] .
$$

Introducing the quantities

$$
\begin{gathered}
E_{a}^{r}[\mathbf{x}]=\frac{\delta \Phi}{\delta \varphi_{a}^{r}(\mathbf{x})}, \\
e_{a}^{r}(t, \mathbf{x})=-V^{-1} \int \mathcal{D}^{2} \varphi E_{a}^{r}\left[\varphi^{1}, \varphi^{2}, t, \mathbf{x}\right],
\end{gathered}
$$

where $V \equiv \int \mathcal{D}^{2} \varphi$, the corresponding velocity is given by

$$
v_{a}^{r}[\mathbf{x}]=u_{a}^{r}[\mathbf{x}]+\rho^{-1}\left(e_{a}^{r}(t, \mathbf{x})+E_{a}^{r}[\mathbf{x}]\right) .
$$

Now we introduce hidden variables $\varphi^{1}(t, \mathbf{x})$ and $\varphi^{2}(t, \mathbf{x})$. The causal evolution of these hidden variables is given by

$$
\frac{\partial \varphi_{a}^{r}(t, \mathbf{x})}{\partial t}=v_{a}^{r}\left[\varphi^{1}, \varphi^{2}, t, \mathbf{x}\right]
$$


where it is understood that the right-hand side is calculated at $\varphi^{r}(\mathbf{x})=\varphi^{r}(t, \mathbf{x})$.

In analogy with ordinary bosonic fields [12], we also introduce the effectivity $e_{n_{P}, n_{A}}$ of the particles guided by the wave function $\psi_{n_{P}, n_{A}}$, given by

$$
e_{n_{P}, n_{A}}\left[\varphi^{1}, \varphi^{2}, t\right]=\frac{\left|\tilde{\Psi}_{n_{P}, n_{A}}\left[\varphi^{1}, \varphi^{2}, t\right]\right|^{2}}{\sum_{n_{P}^{\prime}, n_{A}^{\prime}}\left|\tilde{\Psi}_{n_{P}^{\prime}, n_{A}^{\prime}}\left[\varphi^{1}, \varphi^{2}, t\right]\right|^{2}} .
$$

Since the physical meaning of the effectivity is discussed in detail in Ref. 12 for ordinary bosonic fields, here we only note the final results valid also for the bosonic representation of the fermionic states above. In an ideal experiment in which the number of particles is measured, different $\tilde{\Psi}_{n_{P}, n_{A}}$ functionals do not overlap in the $\left(\varphi^{1}, \varphi^{2}\right)$ space. Consequently, the fields $\varphi^{1}$ and $\varphi^{2}$ necessarily enter one and only one of the "channels" $\tilde{\Psi}_{n_{P}, n_{A}}$. In this case, the effectivity (68) of the corresponding particles is equal to 1, while that of all other particles guided by other wave functions $\psi_{n_{P}^{\prime}, n_{A}^{\prime}}$ is equal to 0 . The effect is the same as if the wave functional $\Psi$ "collapsed" into one of the states $\tilde{\Psi}_{n_{P}, n_{A}}$ with a definite number of particles and antiparticles. This provides a causal description of the creation of particles guided by $\psi_{n_{P}, n_{A}}$ and the destruction of all other particles guided by other wave functions $\psi_{n_{P}^{\prime}, n_{A}^{\prime}}$.

Note that both the corpuscle (particle and antiparticle) positions and the commuting fields $\varphi^{1}, \varphi^{2}$ are the preferred hidden variables that describe the elements of reality associated with fermions. This would not be possible if the corpuscle positions were described by hermitian operators that do not commute with $\varphi^{1}, \varphi^{2}$. However, just as in the relativistic bosonic case [12], there are no hermitian corpuscle-position operators associated with the corpuscle positions. At the level of first-quantization formalism, the absence of the hermitian corpuscle-position operators is a consequence of the fact that the separate sets $\left\{u_{k}(\mathbf{x})\right\}$ and $\left\{v_{k}(\mathbf{x})\right\}$ are not complete, so one cannot construct a $\delta$-function as a linear combination of the functions of only one set. Similarly, at the level of second-quantization formalism, the are no 1-particle states $\left|\Psi^{(P)}\right\rangle$ with the property $\hat{n}^{(P)}(\mathbf{x})\left|\Psi^{(P)}\right\rangle=\delta^{3}(\mathbf{x})\left|\Psi^{(P)}\right\rangle$ (and analogously for 1-antiparticle states). These facts are also related to the already mentioned fact that the conventional theory, in general, does not give definite predictions on the probabilities of corpuscle positions. In our theory, the corpuscle positions correspond to an extension of the conventional theory. As discussed in Sec. 3] the consistency of the probabilistic interpretation of particle and antiparticle wave functions rests on the fact that the probabilities correspond only to an effective statistical description of the underlying deterministic Bohmian trajectories.

\section{CONCLUSION}

In this paper, three new results related to the causal interpretation of fermions have been obtained. First, the usual causal interpretation of the Dirac equation is modified such that it makes a difference between particle and antiparticle trajectories. Second, fermionic interacting QFT is used to construct the multiparticle wave functions that describe particle and antiparticle trajectories in systems with many particles. Third, a causal interpretation of fermionic fields themselves, based on bosonization of fermionic fields, is constructed and used to formulate a causal interpretation of particle creation and destruction.

Besides this progress in the causal interpretation of fermions, a general method of causal interpretation is developed. It can be applied to any quantum system described by a c-number valued wave function $\psi(\vec{\varphi}, t)$ satisfying a Schrödinger equation, provided that the c-number parameters $\varphi_{i}$ are continuous. 
Acknowledgements. The author is grateful to R. Tumulka for valuable comments and discussions and to an anonymous referee for valuable critical remarks that improved the paper. This

work was supported by the Ministry of Science and Technology of the Republic of Croatia under Contract No. 0098002.

\section{References}

[1] D. Bohm, Phys. Rev. 85, 166, 180 (1952).

[2] D. Bohm and B. J. Hiley, Phys. Rep. 144, 323 (1987).

[3] D. Bohm, B. J. Hiley, and P. N. Kaloyerou, Phys. Rep. 144, 349 (1987).

[4] P. R. Holland, Phys. Rep. 224, 95 (1993).

[5] P. R. Holland, The Quantum Theory of Motion (Cambridge University Press, Cambridge, 1993).

[6] P. R. Holland, Found. Phys. 22, 1287 (1992).

[7] C. Dewdney, G. Horton, M. M. Lam, Z. Malik, and M. Schmidt, Found. Phys. 22, 1217 (1992).

[8] P. R. Holland, Phys. Lett. A 128, 9 (1988).

[9] S. Colin, Phys. Lett. A 317, 349 (2003).

[10] J. S. Bell, Phys. Rep. 137, 49 (1986).

[11] J. S. Bell, Speakable and Unspeakable in Quantum Mechanics (Cambridge University Press, Cambridge, 1987).

[12] H. Nikolić, Found. Phys. Lett. 17, 363 (2004).

[13] H. Nikolić, Phys. Lett. B 527, 119 (2002); Erratum 529, 265 (2002).

[14] H. Nikolić, Int. J. Mod. Phys. D 12, 407 (2003).

[15] H. Nikolić, hep-th/0205022, to appear in Gen. Rel. Grav.

[16] R. Floreanini and R. Jackiw, Phys. Rev. D 37, 2206 (1988).

[17] C. Kiefer and A. Wipf, Ann. Phys. 236, 241 (1994).

[18] J. Hallin and P. Liljenberg, Phys. Rev. D 52, 1150 (1995).

[19] J. D. Bjorken and S. D. Drell, Relativistic Quantum Mechanics (McGraw-Hill, New York, 1964).

[20] D. V. Long and G. M. Shore, Nucl. Phys. B 530, 247 (1998).

[21] H. Nikolić, quant-ph/0305131.

[22] R. A. Hyman, S. A. Caldwell, and E. Dalton, quant-ph/0401008. 